Algebraic $8 \mathcal{G}$ Geometric Topology

Volume 3 (2003) 969-992

Published: 4 October 2003

ATG

\title{
Geometric construction of spinors in orthogonal modular categories
}

\author{
AnNA Beliakova
}

\begin{abstract}
A geometric construction of $\mathbb{Z}_{2}$-graded odd and even orthogonal modular categories is given. Their 0 -graded parts coincide with categories previously obtained by Blanchet and the author from the category of tangles modulo the Kauffman skein relations. Quantum dimensions and twist coefficients of 1-graded simple objects (spinors) are calculated. We show that invariants coming from our odd and even orthogonal modular categories admit spin and $\mathbb{Z}_{2}$-cohomological refinements, respectively. The relation with the quantum group approach is discussed.
\end{abstract}

AMS Classification 57M27; 57R56

Keywords Modular category, quantum invariant, Vassiliev-Kontsevich invariant, weight system

\section{Introduction}

In 1993, Lickorish gave a simple geometric construction of 3-manifold invariants based on the Kauffman brackets. The same invariants were obtained earlier by Reshetikhin and Turaev from the representation category of the quantum group $U_{q}\left(\mathfrak{s l}_{2}\right)$. The method of Lickorish was so much easier than the quantum group theoretical one that it inspired many researchers to work on its generalizations.

Recall that a quantum group $U_{q}(\mathfrak{g})$ for any semi-simple Lie algebra $\mathfrak{g}$ and some root of unity $q$ provides 3-manifold invariants. In many cases a representation category of the quantum group is modular (or modularizable), i.e. a functor from the category of 3-cobordisms to the representation category can be constructed (see [8]). This functor is called a Topological Quantum Field Theory (TQFT). 
In order to get a geometric construction of 3-manifold invariants or TQFT's coming from quantum groups of type $A\left(\mathfrak{g}=\mathfrak{s l}_{m}\right)$, a replacement of the Kauffman bracket in Lickorish's approach by the HOMFLY polynomial is needed. This was successfully done in papers of Yokota, Aiston-Morton and Blanchet.

In [4], Blanchet and the author constructed (pre-)modular categories from the category of tangles modulo the Kauffman skein relations. We recovered the invariants of symplectic quantum groups $\left(\mathfrak{g}=\mathfrak{s p}_{m}\right.$ type $C$ ), but only "half" of the invariants for orthogonal groups $\left(\mathfrak{g}=\mathfrak{s o}_{m}\right.$ types $B$ and $\left.D\right)$. Our approach did not provide objects corresponding to spin representations.

In this article we give a geometric construction of two series of orthogonal modular categories which include spinors. We consider the category of framed tangles where colors from the set $\{1,2\}$ are attached to lines. We add the relations given by the kernel of the $\left(\mathfrak{s o}_{m}, V, S\right)$ weight system pulled back by the Vassiliev-Kontsevich invariant. The standard representation $V$ and the spinor representation $S$ are used for 1 -colored and 2-colored lines, respectively. The resulting category admits a natural $\mathbb{Z}_{2}$-grading. The 0 -graded part has the same set of simple objects as the category studied in [4].

We consider two series of parameter specializations for which this 0-graded part is pre-modular. Then we give a recursive construction of idempotents for the $1-$ graded parts of these pre-modular categories. The key point is the observation that encircling any 1-graded object with a line colored with a special 0-graded object, given by a rectangular Young diagram, yields a projector. We calculate quantum dimensions and twist coefficients of 1-graded simple objects (spinors). We show that invariants coming from our odd and even orthogonal modular categories admit spin and $\mathbb{Z}_{2}$-cohomological refinements, respectively.

This paper is organized as follows. In the first section we define the category we will work in. In the second and third sections we construct odd and even orthogonal modular categories. Relations with quantum groups are discussed in the last section.

The author wishes to thank Christian Blanchet for many stimulating discussions. 


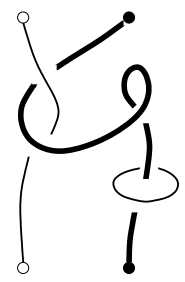

Figure 1: Diagram of a two colored tangle

\section{Basic category}

In this section we define the category which will be studied subsequently. We also analyze its 0-graded part.

\subsection{Category of two colored tangles}

Let us fix an oriented 3-dimensional Euclidean space $\mathbb{R}^{3}$ with coordinates $(x, y, t)$.

Definition 1.1 A two colored tangle $T$ is a 1-dimensional compact smooth sub-manifold of $\mathbb{R}^{3}$ equipped with a normal vector field and lying between two horizontal planes $\{t=a\},\{t=b\}, a>b$, called the top and the bottom planes. The boundary of $T$ lies on two lines $\{t=a, y=0\}$ and $\{t=b, y=0\}$. The normal vector field has coordinates $(0,1,0)$ in boundary points. The map $c$ from the set of connected components of $T$ to the set of colors $\{1,2\}$ is given.

Two colored tangles $T$ and $T^{\prime}$ are equivalent if there is an isotopy sending $T$ to $T^{\prime}$ which respects horizontal planes and colorings.

Connected components of $T$ will be called lines. We represent $T$ by drawing its generic position diagram in blackboard framing. Lines of the second color are drawn bold. An example is given in Figure 1.

An intersection of a two colored tangle with the top and the bottom planes defines a word in the alphabet $\{\circ, \bullet\}$, where $\circ$ and $\bullet$ denote the points of the first and the second color, respectively. For two such words $u$ and $v$, let $(\mathcal{T}, u, v)$ be the set of two colored tangles whose intersection with the top and the bottom planes are given by $u$ and $v$, respectively. 
Definition 1.2 Let $\mathcal{T}$ be the monoidal category whose objects are words in the alphabet $\{\circ, \bullet\}$. For $u, v \in O b(\mathcal{T})$, the set of morphisms $\operatorname{Hom}(u, v)$ from $u$ to $v$ is given by $(\mathcal{T}, u, v)$. The composition of $(\mathcal{T}, u, v)$ with $(\mathcal{T}, v, w)$ is defined by gluing of horizontal planes identifying points corresponding to $v$. Moreover, $u \otimes v:=u v$.

Definition 1.3 Let $f$ be a field. Let $\mathcal{T}_{f}$ be a linearization of $\mathcal{T}$, where formal $f$-linear combinations of tangles are allowed as morphisms. The composition and tensor product are bilinear.

\subsection{Kontsevich integral}

In [9], the category of q-tangles was considered. The objects of this category are non-associative words in the alphabet $\{+,-\}$. The morphisms are framed oriented tangles. It was shown that the universal Vassiliev-Kontsevich invariant extends to a functor from this category to the category of chord diagrams. An analogous construction applies to colored q-tangles.

Let us orient all lines of two colored tangles from the top to the bottom. Let us map a word $u \in O b(\mathcal{T})$ with $n$ letters to the non-associative word of length $n$ in the alphabet $\{+,+\}$ beginning with $n$ left brackets, e.g., $\circ \bullet \circ$ maps to $((++)+)$. This defines a functor from $\mathcal{T}$ into the category of two colored q-tangles. Now the universal Vassiliev-Kontsevich invariant constructed in [9] defines a functor from this category to the category $\mathcal{A}$ of chord diagrams with two colored support. We denote by $Z: \mathcal{T} \rightarrow \mathcal{A}$ the composition.

Let us consider the Lie algebra $\mathfrak{g}=\mathfrak{s o}_{m}$. Let $V$ be the standard and $S$ the spin representation of $\mathfrak{g}$. Let $t \in \mathfrak{g}^{*} \otimes \mathfrak{g}^{*}$ be its Killing form.

Theorem 1.1 There exists a unique $\mathbb{C}$-linear monoidal functor $\mathcal{F}_{\mathfrak{g}, V, S}$ (called weight system) from $\mathcal{A}$ to the category $\operatorname{Mod}_{\mathfrak{g}}$ of the representations of $\mathfrak{g}$ such that it is uniquely characterized by its values on the following elementary morphisms. 


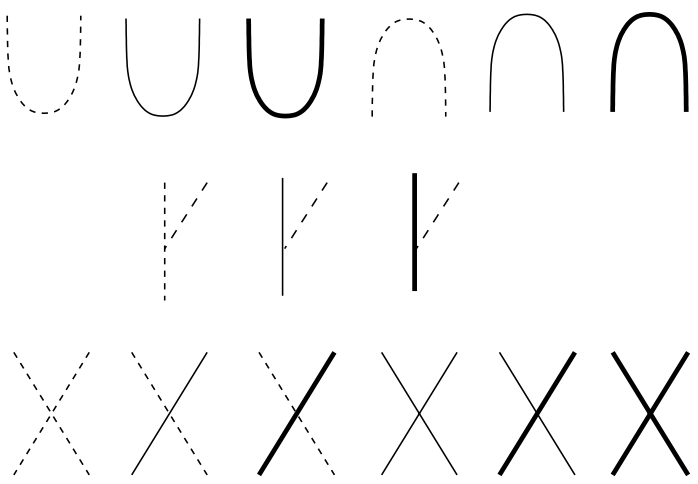

The first three diagrams correspond to the morphisms $\mathfrak{g} \otimes \mathfrak{g} \rightarrow \mathbb{C}, V \otimes V \rightarrow \mathbb{C}$ and $S \otimes S \rightarrow \mathbb{C}$ in $M_{\mathfrak{g}}$ given by the Killing form. The next three are their transposes. The first morphism in the second row is given by the Lie bracket. The next two correspond to the $\mathfrak{g}$-action on $V$ and $S$. The third row describes flips $x \otimes y \mapsto y \otimes x$ in $\mathfrak{g} \otimes \mathfrak{g}, \mathfrak{g} \otimes V, \mathfrak{g} \otimes S, V \otimes V, V \otimes S$ and $S \otimes S$, respectively.

The proof of Bar-Natan [1] can be adapted. An essential point is that the invariant tensors $\varrho(t)$ for any representation $\varrho$ satisfy the classical Yang-Baxter equation, which corresponds to the 4 -term relation in $\mathcal{A}$.

Remark For $\mathfrak{g}=\mathfrak{s o}_{2 n}$, the construction can be modified by orienting 2colored lines and by using the spin representations $S_{ \pm}$in the weight system, according to the orientation.

\subsection{Category $\mathcal{T}_{q}\left(\mathfrak{s o}_{m}\right)$}

The central object of our study is the category $\mathcal{T}_{h}\left(\mathfrak{s o}_{m}\right)$ defined as $\mathcal{T}_{\mathbb{C}}$ modulo the relations given by the kernel of $\mathcal{F}_{\mathfrak{s a}_{m}, V, S}\left(Z\left(\mathcal{T}_{\mathbb{C}}\right)\right)$. The relations are defined a priori over $\mathbb{C}[[h]]$, where $h$ is the formal parameter of the Kontsevich integral. An explicite description of the relations is not known except if we restrict to 1 -colored framed tangles or to 2 -colored ones and use $\mathcal{F}_{\mathfrak{5 0} 7, S}$ weight system. The first case was considered in [10] and the relations are just the Kauffman skein relations. The second case was studied in [12], where a set of relations sufficient to calculate link invariants is given. 
The proof of Le and Murakami in [9] can be used to show that link invariants provided by $\mathcal{T}_{h}\left(\mathfrak{s o}_{m}\right)$ and the quantum group $U_{q}\left(\mathfrak{s o}_{m}\right)$ coincide for odd $m$ if $q=\exp h$ and for even $m$ if $q=\exp 2 h$. The invariant associated by $U_{q}\left(\mathfrak{s o}_{m}\right)$ with a colored link is defined over the ring $R=\mathbb{Q}\left[q^{ \pm \frac{1}{2 D}}\right]$, where $D=2$ if $m$ is odd and $D=4$ for even $m$. $^{*}$ This allows to define $\mathcal{T}_{h}\left(\mathfrak{s o}_{m}\right)$ over $R$ and to use the notation $\mathcal{T}_{q}\left(\mathfrak{s o}_{m}\right)$ for $\mathcal{T}_{h}\left(\mathfrak{s o}_{m}\right)$, where $q=\exp h$ if $m=2 n+1$ and $q=\exp 2 h$ if $m=2 n$.

Let us define a $\mathbb{Z}_{2}$-grading in $\mathcal{T}_{q}\left(\mathfrak{s o}_{m}\right)$ as follows. A grading of $u \in O b\left(\mathcal{T}_{q}\left(\mathfrak{s o}_{m}\right)\right)$ is given by the number of symbols $\bullet$ in $u$ modulo 2 . All morphisms in the category are 0 -graded.

\section{$1.4 \quad 0-$ graded idempotents}

Let $u \in O b\left(\mathcal{T}_{q}\left(\mathfrak{s o}_{m}\right)\right)$. A nonzero morphism $T \in \operatorname{End}_{\mathcal{T}_{q}\left(\mathfrak{s o}_{m}\right)}(u)$ is called a minimal idempotent if $T^{2}=T$ and for any $X \in \operatorname{End}_{\mathcal{T}_{q}\left(\mathfrak{s o}_{m}\right)}(u)$ there exists a constant $c \in R$, such that $T X T=c T$. A standard procedure called idempotent completion allows to add idempotents as objects into the category. Objects given by minimal idempotents are called simple. The idempotent completion of $\mathcal{T}_{q}\left(\mathfrak{s o}_{m}\right)$ is denoted by the same symbol. Let us equip $\mathcal{T}_{q}\left(\mathfrak{s o}_{m}\right)$ with a direct sum of objects in a formal way.

In [5], we gave a geometric construction of minimal idempotents in the category of (framed non-oriented) tangles modulo the Kauffman skein relations.

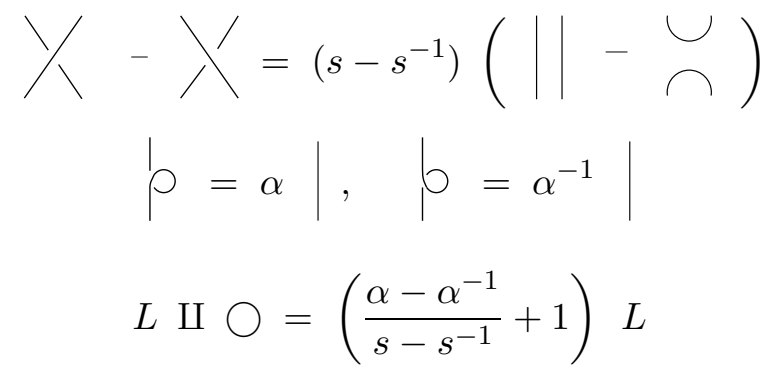

Figure 2: Kauffman relations

The idempotents were numbered by integer partitions or Young diagrams $\lambda=$

\footnotetext{
${ }^{*}$ The integrality result of T. Le shows that even a smaller ring can be considered.
} 
$\left(\lambda_{1} \geq \lambda_{2} \geq \ldots \geq \lambda_{k}\right)$. Their twist coefficients and quantum dimensions were calculated.

Lemma 1.2 After the substitution $\alpha=s^{m-1}, s=\exp h$, the idempotents constructed in [5] give the whole set of minimal idempotents of the 0-graded part of $\mathcal{T}_{h}\left(\mathfrak{s o}_{m}\right)$.

Proof Let us first consider 1-colored tangles. The set of relations in $\mathcal{T}_{h}\left(\mathfrak{s o}_{m}\right)$ for 1-colored tangles coincides with the Kauffman skein relations, where $\alpha=$ $s^{m-1}, s=\exp h$ (see [10] for the proof, - attention, - Le and Murakami use a different normalization for the trivial knot). Minimal idempotents for tangles modulo the Kauffman skein relations are constructed in [5].

From the representation theory of the classical orthogonal Lie algebras (see e.g. [7] p. 291-296) we know that $S \otimes S$ decomposes into a direct sum of simple objects numbered by integer partitions. This implies that the addition of an even number of 2-colored lines to 1 -colored tangles does not create new minimal idempotents.

In [4], we found seven series of specializations of parameters $\alpha$ and $s$, such that the category of tangles modulo the Kauffman skein relations with these specializations becomes pre-modular (after idempotent completion and quotienting by negligible morphisms). In all cases, $s$ is a root of unity and $\alpha$ is a power of $s$. The specializations $\alpha=s^{2 n}, s^{4 n+4 k}=1$ and $\alpha=s^{2 n-1}, s^{4 n+4 k-4}=1$ lead to odd and even orthogonal categories $B^{n,-k}$ and $D^{n, k}$, respectively.

In the remainder of the paper we will complete $B^{n,-k}$ and $D^{n, k}$ with 1 -graded simple objects. The odd and even orthogonal cases will be treated separately.

\section{Odd orthogonal modular categories}

This section is devoted to the construction of odd orthogonal modular categories. We show that these categories lead to spin TQFT's and calculate spin Verlinde formulas. 


\section{$2.1 \quad 0$-graded objects}

Let us use the standard notation $B_{n}$ for $\mathfrak{s o}_{2 n+1}$. We fix a primitive $(4 n+4 k)$ th root of unity $q$ and choose $v$ with $v^{2}=q$. In this specialization, the set of 0-graded simple objects of $\mathcal{T}_{q}\left(B_{n}\right)$ (modulo negligible morphisms) is given by Young diagrams (or integer partitions) from the set

$$
\tilde{\Gamma}=\left\{\lambda: \lambda_{1}+\lambda_{2} \leq 2 k+1, \lambda_{1}^{\vee}+\lambda_{2}^{\vee} \leq 2 n+1\right\}
$$

(see [4] p. 487 for the proof, put $s=q$ ). Here $\lambda_{i}^{\vee}$ denotes the number of cells in the $i$ th column of $\lambda$.

The set $\tilde{\Gamma}$ admits an algebra structure with the multiplication given by the tensor product and the addition given by the direct sum. The empty partition is the one in this algebra and it will be denoted by 1 . (Please not confuse with the partition $V=(1)$ corresponding to the object $\circ$.)

The set $\tilde{\Gamma}$ has two invertible objects of order two. The object $J=(2 k+1)$, given by the one row Young diagram with $2 k+1$ cells in it, and the object $1^{2 n+1}$ given by the one column Young diagram with $2 n+1$ cells in it. They are 0 -transparent, i.e. they have trivial braiding with any other 0 -graded object. The tensor square of each of them is the trivial object. The twist coefficient of $J$ is minus one and of $1^{2 n+1}$ is one. Let us consider the following set

$$
\Gamma_{0}=\left\{\lambda: \lambda_{1}+\lambda_{2} \leq 2 k+1, \lambda_{1}^{\vee} \leq n\right\} .
$$

Any $\lambda \in \tilde{\Gamma}$ is either contained in $\Gamma_{0}$ or is isomorphic to $1^{2 n+1} \otimes \mu$ with $\mu \in \Gamma_{0}$, where $1^{2 n+1} \otimes \mu$ and $\mu$ are both simple with the same quantum dimensions, braiding and twist coefficients. There exists a standard procedure called modularization (or modular extension), which allows to path to a new category, where $1^{2 n+1} \otimes \mu$ and $\mu$ are identified. We will denote by $\mathcal{T}_{q}\left(\tilde{B}_{n}\right)$ this new category. Its set of simple 0 -graded objects is $\Gamma_{0}$. The existence criterion for such modularization functors was developed by Bruguières. In [4], a geometric construction of these functors is given.

\subsection{Recursive construction of 1-graded idempotents}

The rectangular 0 -graded object $A=k^{n} \in \Gamma_{0}$, consisting of $n$ rows with $k$ cells in each, plays a key role in our construction of 1-graded idempotents. Let us define $P_{ \pm}=\frac{1}{2}(1 \pm A)$. 
Let us enumerate 1-graded simple objects by partitions consisting of $n$ nonincreasing half-integers. The partition $S=(1 / 2, \ldots, 1 / 2)$ is used for the object - ${ }^{\dagger}$ The first step in the recursive construction of minimal 1-graded idempotents is given by the following proposition.

Proposition 2.1 Let $\lambda=\left(\lambda_{1}\right)$ be a one row Young diagram with $1 \leq \lambda_{1} \leq 2 k$. The tangles

$$
\tilde{P}_{+}(\lambda)=\frac{\lambda||_{-l}}{\mid \downarrow} P_{+} \quad \tilde{P}_{-}(\lambda)=\frac{\lambda \mid\rfloor_{-l}}{\mid \downarrow} P_{-}
$$

are minimal idempotents projecting into simple objects $\left(\lambda_{1}+1 / 2,1 / 2, \ldots, 1 / 2\right)$ and $\left(\lambda_{1}-1 / 2,1 / 2, \ldots, 1 / 2\right)$. Here $\tilde{P}_{+}(\lambda)$ projects into the first partition if $\lambda_{1}=0$ $\bmod 2$, otherwise into the second.

Proof Let us decompose the identity of $\lambda \otimes S$ as follows.

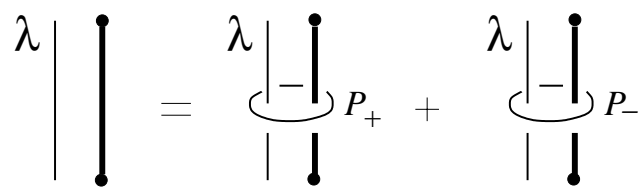

From the representation theory of $B_{n}$ we know that

$$
\lambda \otimes S=\left(\lambda_{1}+1 / 2,1 / 2, \ldots, 1 / 2\right) \oplus\left(\lambda_{1}-1 / 2,1 / 2, \ldots, 1 / 2\right) .
$$

Therefore, $\operatorname{dim} \operatorname{End}_{\mathcal{T}_{q}\left(\tilde{B}_{n}\right)}(\lambda \otimes S)$ is maximal two. Note that $J \otimes S$ is simple, because $J=(2 k+1)$ is invertible. Using the equality of the colored link invariants in $\mathcal{T}_{q}\left(\tilde{B}_{n}\right)$ and $U_{q}\left(B_{n}\right)$, semi-simplicity of the modular category for $U_{q}\left(B_{n}\right)$ and Lemma 5.1 in the Appendix, we see that

$$
\tilde{P}_{ \pm}(\lambda) \tilde{P}_{ \pm}(\lambda)=\tilde{P}_{ \pm}(\lambda) \quad \tilde{P}_{ \pm}(\lambda) \tilde{P}_{\mp}(\lambda)=0
$$

for any $\lambda \in \Gamma_{0}$. Furthermore, these morphisms are non-negligible. The claim follows.

Remark 2.2 In the proof we use the quantum group formulas for the quantum dimension and the $S$-matrix. These formulas can also be obtained by applying an appropriate weight system to the Kontsevich integral of the unknot and of the Hopf link, which were calculated recently in [2]. This will make our approach completely independent from the quantum group theoretical one.

${ }^{\dagger}$ The relation between such partitions and dominant weights of $B_{n}$ is explained in the appendix. 
Let $\lambda=\left(\lambda_{1}, \ldots, \lambda_{p}\right)$ be a Young diagram with $\lambda_{1}+\lambda_{2} \leq 2 k$ and $2 \leq p \leq n$. Let $\nu$ be obtained by removing one cell from the last row of $\lambda$. Let us assume per induction that we can construct an idempotent $p_{\mu}^{\nu S}$ projecting $\nu \otimes S$ into a simple component $\mu$. We know from the classical theory that the tensor product $\lambda \otimes S$ decomposes into simple objects as follows:

$$
\lambda \otimes S=\oplus\left(\lambda_{1} \pm 1 / 2, \lambda_{2} \pm 1 / 2, \ldots, \lambda_{p} \pm 1 / 2,1 / 2, \ldots, 1 / 2\right)
$$

Contributions not corresponding to non-increasing partitions do not appear in this decomposition. A quasi-idempotent $\tilde{p}_{\mu}^{\lambda S}$ projecting to a partition $\mu$ from the set $I=\left\{\left(\lambda_{1} \pm 1 / 2, \lambda_{2} \pm 1 / 2, \ldots, \lambda_{p}-1 / 2,1 / 2, \ldots, 1 / 2\right)\right\}$ can be obtained as follows:

$$
\left(\tilde{y}_{\lambda} \otimes i d_{S}\right)\left(i d_{|\lambda|-1} \otimes \tilde{P}_{+}\right) p_{\mu}^{\nu S}\left(i d_{|\lambda|-1} \otimes \tilde{P}_{+}\right)\left(\tilde{y}_{\lambda} \otimes i d_{S}\right) .
$$

Here where $\tilde{y}_{\lambda}$ is the 0 -graded minimal idempotent defined in [5], $\tilde{P}_{+}$is the idempotent $p_{S}^{V S}$ given by encircling the 2-colored line and the 1 -colored line starting from the last cell in the last row of $\lambda$ with a line colored by $P_{+}$. Normalizing (if necessary) this quasi-idempotent we get $p_{\mu}^{\lambda S}$. The projector onto $\left(\lambda_{1}+1 / 2, \ldots, \lambda_{p}+1 / 2,1 / 2, \ldots, 1 / 2\right)$ is given by $\tilde{y}_{\lambda} \otimes i d_{S}-\sum_{\mu \in I} p_{\mu}^{\lambda S}$. It remains to show that $p_{\mu}^{\lambda S}$ is not negligible. The trace of the morphism $\left(\tilde{y}_{(1,1)} \otimes\right.$ $\left.i d_{S}\right)\left(i d_{V} \otimes \tilde{P}_{+}\right)$is nonzero. Here we use that $\operatorname{dim} S \neq 0$ (see next subsection). Analogously, the trace of $\left(i d_{V} \otimes \tilde{P}_{+}\right)\left(\tilde{y}_{(2)} \otimes i d_{S}\right)$ is nonzero. Therefore, $p_{\mu}^{\lambda S}$ is a composition of non-negligible morphisms.

As a result, we can construct 1-graded simple objects numbered by partitions consisting of $n$ non-increasing half-integers from the set

$$
\Gamma=\left\{\lambda: \lambda_{1}+\lambda_{2} \leq 2 k+1\right\} .
$$

We hope to be able to prove the following statement in the future.

Conjecture 2.3 Let $\lambda=\left(\lambda_{1}, \ldots, \lambda_{p}\right)$ be a Young diagram with $\lambda_{1}+\lambda_{2} \leq 2 k$, $p \leq n$. For $b=\left(\lambda_{1}+1 / 2, \ldots, \lambda_{p}+1 / 2,1 / 2, \ldots, 1 / 2\right)$ we have

$$
p_{b}^{\lambda S}=\left(\tilde{y}_{\lambda} \otimes i d_{S}\right)\left(\tilde{P}_{1}\left(\lambda_{1}\right) \otimes i d\right) \ldots\left(i d \otimes \tilde{P}_{p}\left(\lambda_{p}\right)\right)\left(\tilde{y}_{\lambda} \otimes i d_{S}\right),
$$

where

$$
\tilde{P}_{i}\left(\lambda_{i}\right)=\left\{\begin{array}{llll}
\tilde{P}_{+}\left(\lambda_{i}\right) & : & \lambda_{i}=0 & \bmod 2 \\
\tilde{P}_{-}\left(\lambda_{i}\right) & : & \lambda_{i}=1 & \bmod 2
\end{array}\right.
$$

Algebraic 83 Geometric Topology, Volume 3 (2003) 
An example of such projection onto $(7 / 2,5 / 2,3 / 2)$ for $\lambda=(3,2,1)$ is drawn below.

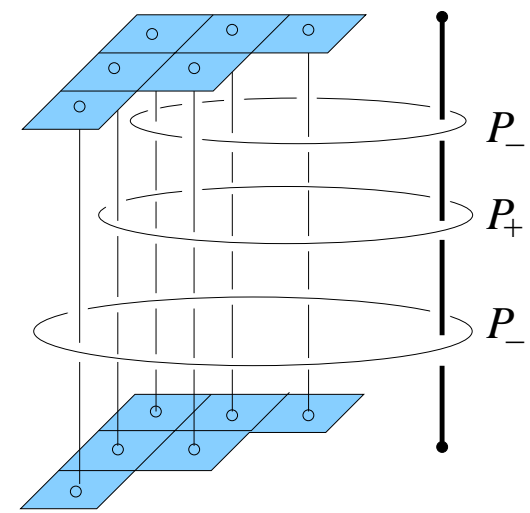

\subsection{Quantum dimensions.}

By applying the $\left(B_{n}, S\right)$ weight system to the Kontsevich integral of the 2colored unknot we get ${ }^{\ddagger}$

$$
\operatorname{dim} S=\left(v+v^{-1}\right)\left(v^{3}+v^{-3}\right) \ldots\left(v^{2 n-1}+v^{-2 n+1}\right)
$$

Here $q=v^{2}$. By closing (1) with $\lambda=(1)$ we get $\operatorname{dim} V \operatorname{dim} S=\operatorname{dim} S+\operatorname{dim} X$, which allows to calculate $\operatorname{dim} X$. We conclude that it is given by formula (7) in the Appendix with $\lambda=(3 / 2,1 / 2, \ldots, 1 / 2)$.

Proposition 2.4 The quantum dimension of a simple object $\lambda \in \Gamma$ is given by formula (7).

Proof For integer partitions, the claim was proved in [4]. In fact, (7) coincides with the formula given in Proposition 3.3 of [4]. Let us assume per induction that the quantum dimensions of 1-graded simple objects are given by this formula. We are finished if we can show that for a $p$ row Young diagram $\lambda$

$$
\operatorname{dim} S \operatorname{dim} \lambda=\sum_{\tilde{s} \in \mathbb{Z}_{2}^{p}} \operatorname{dim}(\lambda+\tilde{s}),
$$

where $\mathbb{Z}_{2}^{p}=\{( \pm 1 / 2, \ldots, \pm 1 / 2,1 / 2, \ldots, 1 / 2)\}$. Note that if $\lambda_{i}=\lambda_{i+1}$,

$$
\operatorname{dim}\left(\lambda_{1} \pm \frac{1}{2}, \ldots, \lambda_{i}-\frac{1}{2}, \lambda_{i}+\frac{1}{2}, \ldots, \lambda_{n} \pm \frac{1}{2}\right)=0,
$$

\footnotetext{
${ }^{\ddagger}$ This computation will be published elsewhere.
} 
because terms in (7) corresponding to $w$ and $\sigma_{i} w$ cancel with each other. Here $\sigma_{i}$ interchanges the $i$ th and $(i+1)$ th coordinates. Using

$$
\operatorname{dim} S=\sum_{\tilde{s} \in \mathbb{Z}_{2}^{n}} v^{2(\tilde{s} \mid \rho)}
$$

we get

$$
\begin{aligned}
\operatorname{dim} S \operatorname{dim} \lambda & =\frac{1}{\psi} \sum_{w \in W, \tilde{s} \in \mathbb{Z}_{2}^{n}} s n(w) v^{2\left(w^{-1}(\lambda+\rho)+\tilde{s} \mid \rho\right)} \\
& =\frac{1}{\psi} \sum_{w \in W, w^{\prime} \in \mathbb{Z}_{2}^{n}} s n(w) v^{2\left(w^{-1}(\lambda+\rho)+w^{-1} w^{\prime}(S) \mid \rho\right)} \\
& =\frac{1}{\psi} \sum_{w^{\prime} \in \mathbb{Z}_{2}^{n}} \sum_{w \in W} s n(w) v^{2\left(\lambda+\rho+w^{\prime}(S) \mid w(\rho)\right)} \\
& =\sum_{\tilde{s} \in \mathbb{Z}_{2}^{p}} \operatorname{dim}(\lambda+\tilde{s})
\end{aligned}
$$

\subsection{Twist coefficients}

Let us denote by $t_{\mu}$ the twist coefficient of the simple object $\mu$. Then by twisting (2) we have the following identity

$$
\left.t_{\lambda} t_{S}{ }^{\lambda}\right)^{s}=\sum_{\tilde{s} \in \mathbb{Z}_{2}^{p}} t_{\lambda+\tilde{s}}^{\lambda} \nearrow^{\lambda}{ }^{s+\tilde{s}} .
$$

Replacing the positive twist with the negative one we get a similar identity involving inverse twist coefficients. By closing the $\lambda$-colored line in these two identities we obtain

$$
\begin{gathered}
t_{\lambda} t_{S} \bigodot_{\mid}^{S}=\sum_{\tilde{s}} t_{\lambda+\tilde{s}} \frac{\operatorname{dim}(\lambda+\tilde{s})}{\operatorname{dim} S} \mid \\
t_{\lambda}^{-1} t_{S}^{-1} \bigodot_{\mid}^{S}=\sum_{\tilde{s}} t_{\lambda+\tilde{s}}^{-1} \frac{\operatorname{dim}(\lambda+\tilde{s})}{\operatorname{dim} S} \mid .
\end{gathered}
$$

Algebraic 63 Geometric Topology, Volume 3 (2003) 
This implies the following formula:

$$
\sum_{\tilde{s} \in \mathbb{Z}_{2}^{n}}\left(t_{S}^{-1} t_{\lambda}^{-1} t_{\lambda+\tilde{s}}-t_{S} t_{\lambda} t_{\lambda+\tilde{s}}^{-1}\right) \operatorname{dim}(\lambda+\tilde{s})=0
$$

Using (3) and the formulas for the quantum dimension we can calculate twist coefficients of simple 1-graded objects recursively. Note that $t_{S}=v^{n^{2}+n / 2}$ is determined by the action of the Casimir on the spin representation.

Proposition 2.5 The twist coefficient of a simple object $\mu \in \Gamma$ is given by the following formula:

$$
t_{\mu}=v^{(\mu+2 \rho \mid \mu)}
$$

Proof In [4] it was shown that the twist coefficients of 0 -graded objects are given by this formula. Now let us assume per induction that this formula holds for 1-graded objects. We are finished if we can prove (3) with this induction hypothesis.

Substituting (4) and quantum dimensions in (3), we get:

$$
\begin{array}{r}
v^{-(4 \rho \mid s)} \sum_{\tilde{s} \in \mathbb{Z}_{2}^{n}} \sum_{w \in W} s n(w) v^{2(\lambda+\tilde{s}+\rho \mid w(\rho))} v^{2(\lambda+\rho \mid \tilde{s})} \\
=\sum_{\tilde{s} \in \mathbb{Z}_{2}^{n}} \sum_{w \in W} s n(w) v^{2(\lambda+\tilde{s}+\rho \mid w(\rho))} v^{-2(\lambda+\rho \mid \tilde{s})}
\end{array}
$$

Using the fact that the Weyl group $W$ is a semi-direct product of the symmetric group $S_{n}$ and $\mathbb{Z}_{2}^{n}$ (acting on $\mathbb{R}^{n}$ by changing signs of coordinates), we write $w=g^{\prime} \sigma$ and $\tilde{s}=g(S)$ with $g, g^{\prime} \in \mathbb{Z}_{2}^{n}$ and $\sigma \in S_{n}$. With this notation, (5) follows from the following two identities:

$$
\begin{gathered}
\sum_{g, g^{\prime} \in \mathbb{Z}_{2}^{n}, \sigma \in S_{n}} s n\left(g^{\prime} \sigma\right) v^{2\left(\lambda+\rho \mid g^{\prime} \sigma(\rho)+g(S)\right)} v^{ \pm 2\left(g^{\prime} g(S)-S \mid \sigma(\rho)\right)} \\
=\sum_{g \in \mathbb{Z}_{2}^{n}, \sigma \in S_{n}} s n(g \sigma) v^{2(g(\lambda+\rho) \mid \sigma(\rho)+S)}
\end{gathered}
$$

To get the second one we replace $\tilde{s}$ by $-\tilde{s}$. In the rest of the proof we will show (6). The idea is that terms with $g \neq g^{\prime}$ cancel in pairs. Let us first consider the simplest case, when $g^{\prime}(x)$ differs from $g(x)$ only by a sign of the $i$ th coordinate. We write $g^{\prime}=g_{i} g$. Let us denote by $t$ the $i$ th half-integer coordinate of $\sigma(\rho)$, i.e. $(\sigma(\rho))_{i}=t$. Then there are two possibilities: (a) there 
exists $j$ with $(\sigma(\rho))_{j}=t-1$ or (b) $t=1 / 2$. In the first case, we put $\tilde{\sigma}=\sigma_{i j} \sigma$ and $\tilde{g}=g_{i} g_{j} g$, where $\sigma_{i j}$ interchange the $i$ th and $j$ th coordinates. Analyzing the four possibilities $g\left(x_{i}\right)= \pm x_{i}, g\left(x_{j}\right)= \pm x_{j}$, we see that

$$
g^{\prime} \sigma(\rho)+g(S)=g^{\prime} \tilde{\sigma}(\rho)+\tilde{g}(S) .
$$

The claim then follows from the fact that $\operatorname{sn}\left(g^{\prime} \sigma\right)=-\operatorname{sn}\left(g^{\prime} \tilde{\sigma}\right)$ and $\left(g^{\prime} g(S)-\right.$ $S \mid \sigma(\rho))=\left(g^{\prime} \tilde{g}(S)-S \mid \tilde{\sigma}(\rho)\right)$.

In case (b), we put $\tilde{g}^{\prime}=g_{i} g^{\prime}, \tilde{g}=g_{i} g$ and $\tilde{\sigma}=\sigma$. Case by case checking shows that terms in (6) corresponding to $g, g^{\prime}, \sigma$ and $\tilde{g}, \tilde{g}^{\prime}, \tilde{\sigma}$ cancel with each other. Note that if $g^{\prime}(x)$ and $g(x)$ are different for all $n$ coordinates, then we can proceed as in case (b).

Let us assume that $g^{\prime}(x)$ and $g(x)$ differs in less than $n$ coordinates. Then there exists $j$ with $(\sigma(\rho))_{j}=1 / 2$. If $g\left(x_{j}\right)=-g^{\prime}\left(x_{j}\right)$, then we finish with (b), if not, we compare $g^{\prime}\left(x_{i}\right)$ and $g\left(x_{i}\right)$ with $(\sigma(\rho))_{i}=3 / 2,5 / 2, \ldots$ Proceeding in this way we will find a pair of indices $i, j$, such that $(\sigma(\rho))_{i}-(\sigma(\rho))_{j}=1$, $g\left(x_{j}\right)=g^{\prime}\left(x_{j}\right)$, but $g\left(x_{i}\right)=-g^{\prime}\left(x_{i}\right)$. Then we continue as in case (a).

\subsection{Modular category $B_{n}^{k}$}

The previous results imply that the category $\mathcal{T}_{q}\left(\tilde{B}_{n}\right)$ defined on a $(4 n+4 k)$ th root of unity $q$ is pre-modular. Its simple objects are numbered by integer or half-integer partitions $\lambda=\left(\lambda_{1}, \ldots, \lambda_{n}\right)$ with $\lambda_{1} \geq \ldots \geq \lambda_{n} \geq 0$ from $\Gamma=\{\lambda$ : $\left.\lambda_{1}+\lambda_{2} \leq 2 k+1\right\}$. Let us call this category $B_{n}^{k}$.

Theorem 2.6 The category $B_{n}^{k}$ is modular.

Proof It remains to prove that $B_{n}^{k}$ has no nontrivial transparent objects. Let $b=\left(b_{1}+1 / 2, b_{2}+1 / 2, \ldots, b_{n}+1 / 2\right)$ be a 1 -graded simple object. The object $J \otimes b$ is simple and is given by partition $b^{\prime}=\left(2 k+1-b_{1}-1 / 2, b_{2}+1 / 2, \ldots, b_{n}+1 / 2\right)$. This is because $J$ is invertible and $b^{\prime}$ is the only object in $\Gamma$ with the correct twist coefficient and quantum dimension. It follows

$$
\left.t_{J} t_{b}\right)_{1}^{b^{\prime}}=t_{b^{\prime}}
$$

Algebraic 83 Geometric Topology, Volume 3 (2003) 
Inserting twist coefficients we obtain that the braiding coefficient of $J$ and $b$ is $(-1)$. This implies that $J$ is not transparent in $B_{n}^{k}$ and that no 1 -graded simple object can be transparent. But the 0 -graded part of $B_{n}^{k}$ has not even a further nontrivial 0 -transparent object.

\subsection{Refinements}

It was shown by Blanchet in [6] that any modular category with an invertible object $J$ of order 2 (i.e. $J^{2}=1$ ), whose twist coefficient is $(-1)$ and quantum dimension is 1 , provides invariants of 3-manifolds equipped with spin structure. The $\mathbb{Z}_{2}$-grading defined in [6] by means of $J$ coincides with the one used in this paper. The Kirby color decomposes as $\Omega=\Omega_{0}+\Omega_{1}$ according to this grading. The invariants of closed 3 -manifolds equipped with spin structure are defined by putting the 1-graded Kirby color on the components of a surgery link belonging to the so-called characteristic sublink (defined by the spin structure) and the 0 graded Kirby color on the other components. The ordinary 3-manifold invariant decomposes into a sum of refined invariants over all spin structures. A spin TQFT can also be constructed (see e.g. [3]). It associates a vector space $V\left(\Sigma_{g}, \mathrm{~s}\right)$ to a genus $g$ surface $\Sigma_{g}$ with spin structure s.

Proposition 2.7 The category $B_{n}^{k}$ provides a spin TQFT. Furthermore,

$$
\begin{aligned}
\frac{4^{g}}{\langle\Omega\rangle^{g-1}} \operatorname{dim} V\left(\Sigma_{g}, \mathrm{~s}\right)= & \sum_{\lambda \in \Gamma \backslash \Gamma_{1}}(\operatorname{dim} \lambda)^{2-2 g} \\
& +(-1)^{\operatorname{Arf}(\mathbf{s})} 2^{g} \sum_{\lambda \in \Gamma_{1}}(\operatorname{dim} \lambda)^{2-2 g}
\end{aligned}
$$

where $\langle\Omega\rangle$ is the invariant of the Kirby-colored unknot, $\operatorname{Arf}(\mathrm{s})$ is the Arf invariant and $\Gamma_{1}=\left\{\lambda \in \Gamma: \lambda_{1}=k+1 / 2\right\}$.

Proof A spin Verlinde formula was computed by Blanchet in [6, Theorem 3.3]. It uses the action of $J$ on $\Gamma$ given by the tensor product. In our case,

$$
J \otimes\left(\lambda_{1}, \lambda_{2}, \ldots, \lambda_{n}\right)=\left(2 k+1-\lambda_{1}, \lambda_{2}, \ldots, \lambda_{n}\right)
$$

(compare [4] and the proof of Theorem 2.6.)

Therefore, there are only two different cases. If $\lambda_{1} \neq k+1 / 2$, then $\#$ or $b(\lambda)=2$, $|\operatorname{Stab}(\lambda)|=1$. If $\lambda_{1}=k+1 / 2$, then $\# \operatorname{orb}(\lambda)=1,|\operatorname{Stab}(\lambda)|=2$. The result follows by the direct application of the Blanchet formula. 


\section{Even orthogonal modular categories}

In this section we construct even orthogonal modular categories. We show that corresponding invariants admit cohomological refinements and calculate the refined Verlinde formulas.

\section{$3.1 \quad 0$-graded objects}

Let us use the standard notation $D_{n}$ for $\mathfrak{s o}_{2 n}$. We fix a primitive $(2 k+2 n-2)$ th root of unity $q$ and $v$ with $v^{2}=q$. According to [4], the category $\mathcal{T}_{q}\left(D_{n}\right)$ has the following set of 0 -graded simple objects

$$
\tilde{\Gamma}=\left\{\lambda: \lambda_{1}+\lambda_{2} \leq 2 k, \lambda_{1}^{\vee}+\lambda_{2}^{\vee} \leq 2 n\right\} .
$$

This set contains two invertible objects of order two: $1^{2 n}$ and $2 k$. They are 0 -transparent, with twist coefficients and quantum dimensions are equal to 1 . This implies that the 0 -graded part of $\mathcal{T}_{q}\left(D_{n}\right)$ is modularizable. After modular extension by the group generated by $1^{2 n}$ we get a new category, which will be denoted by $\mathcal{T}_{q}\left(\tilde{D}_{n}\right)$. The objects $1^{2 n} \otimes \nu$ and $\nu$ are isomorphic there for any $\nu \in \tilde{\Gamma}$. The 0 -graded objects $\lambda$ with $\lambda_{1}^{\vee}=n$ do not remain simple in $\mathcal{T}_{q}\left(\tilde{D}_{n}\right)$ and decompose as $\lambda=\lambda_{-}+\lambda_{+}$. The objects $\lambda_{ \pm}$have the same quantum dimensions and twist coefficients. We will use the partitions $\left(\lambda_{1}, \ldots, \pm \lambda_{n}\right)$ for $\lambda_{ \pm}$. The set of 0 -graded simple objects of $\mathcal{T}_{q}\left(\tilde{D}_{n}\right)$ is

$$
\Gamma_{0}=\left\{\lambda: \lambda_{1}+\lambda_{2} \leq 2 k, \lambda_{1}^{\vee}<n\right\} \cup\left\{\lambda_{ \pm}: \lambda_{1}+\lambda_{2} \leq 2 k, \lambda_{1}^{\vee}=n\right\} .
$$

Let $A_{+}$be the simple 0 -graded object of $\mathcal{T}_{q}\left(\tilde{D}_{n}\right)$ obtained after splitting of $A=$ $(k, k, \ldots k)=k^{n}$. Let $i=v^{n+k-1}$ and $P_{ \pm}=\frac{1}{2}\left(1 \pm(-i)^{n} A_{+}\right)$. Then analogously to the odd orthogonal case, encircling of a spinor $b=\left(\frac{2 b_{1}+1}{2}, \ldots, \frac{2 b_{n}+1}{2}\right)$ by $P_{+}$ gives the identity morphism if $\sum_{i} b_{i}=0 \bmod 2$ and is zero otherwise. The proof is given in the appendix.

\subsection{Recursive construction of 1-graded idempotents}

The group $D_{n}$ has two spin representations $S_{ \pm}$given by the highest weights $(1 / 2, \ldots, \pm 1 / 2)$. In order to distinguish them we put an orientation on the $2-$ colored lines. 
Let $w \in \mathbb{Z}_{2}^{p}$ act on coordinates of $\mathbb{R}^{p}$ by sign changing. We put $s n(w)=1$ if it changes the signs of an even number of coordinates and $s n(w)=-1$ otherwise. Let $s=(1 / 2, \ldots, 1 / 2) \in \mathbb{R}^{p}$. For any highest weight $\lambda=\left(\lambda_{1}, \ldots, \lambda_{p}, 0, \ldots, 0\right)$, the tensor product $\lambda \otimes S_{ \pm}$decomposes in $D_{n}$ as follows:

$$
\lambda \otimes S_{ \pm}=\bigoplus_{w \in \mathbb{Z}_{2}^{p}}\left(\lambda_{1}+w\left(s_{1}\right), \ldots, \lambda_{p}+w\left(s_{p}\right), 1 / 2, \ldots, \pm s n(w) 1 / 2\right)
$$

If $p=n$, we have

$$
\lambda \otimes S_{ \pm}=\bigoplus_{w \in \mathbb{Z}_{2}^{n-1}}\left(\lambda_{1}+w\left(s_{1}\right), \lambda_{2}+w\left(s_{2}\right), \ldots, \lambda_{n} \pm \operatorname{sn}(w) 1 / 2\right) .
$$

In particular,

$$
V \otimes S_{+}=S_{-}+(3 / 2,1 / 2, \ldots, 1 / 2), \quad V \otimes S_{-}=S_{+}+(3 / 2,1 / 2, \ldots,-1 / 2) .
$$

The corresponding idempotents are given by $\tilde{P}_{ \pm}(V)$.

Suppose that we can decompose $\nu \otimes S_{ \pm}$into simple objects if $\nu$ is obtained by removing one cell from the last row of $\lambda$. Then the projection $p_{\mu}^{\lambda S_{+}}$to $\mu \subset \lambda \otimes S_{+}\left(\right.$with $\left.\mu \neq h=\left(\lambda_{1}+1 / 2, \ldots, \lambda_{k}+1 / 2,1 / 2, \ldots, 1 / 2\right)\right)$ can be obtained by normalizing the following morphism

$$
\left(\tilde{y}_{\lambda} \otimes i d_{S_{+}}\right)\left(i d_{|\lambda|-1} \otimes \tilde{P}_{+}\right) p_{\mu}^{\nu S_{-}}\left(i d_{|\lambda|-1} \otimes \tilde{P}_{+}\right)\left(\tilde{y}_{\lambda} \otimes i d_{S_{+}}\right),
$$

where $\tilde{P}_{+}$is given by encircling the 2 -colored line and the 1 -colored line starting from the last cell in the last row of $\lambda$ with a line colored by $P_{+}$. The idempotent $p_{h}^{\lambda S_{+}}$is given by $\tilde{y}_{\lambda} \otimes i d_{S_{+}}-\sum_{\mu} p_{\mu}^{\lambda S_{+}}$. The case $\lambda \otimes S_{-}$is similar.

\subsection{Modular category $D_{n}^{k}$}

Analogously to the odd orthogonal case, one can show that the quantum dimensions of spinors are given by the formula (7) and the twist coefficient of a simple object $\mu$ of $\mathcal{T}_{q}\left(\tilde{D}_{n}\right)$ is $v^{(\mu+2 \rho \mid \mu)}$.

We conclude that the category $\mathcal{T}_{q}\left(\tilde{D}_{n}\right)$ at a $(2 n+2 k-2)$ th root of unity $q$ is pre-modular. Its simple objects are given by integer or half-integer partitions $\lambda=\left(\lambda_{1}, \ldots, \lambda_{n-1}, \pm \lambda_{n}\right)$ with $\lambda_{1} \geq \lambda_{2} \geq \ldots \geq \lambda_{n} \geq 0$ from the set $\Gamma=\{\lambda$ : $\left.\lambda_{1}+\lambda_{2} \leq 2 k\right\}$. Let us denote this category by $D_{n}^{k}$. Taking into account that the object $2 k$ has the braiding coefficient $(-1)$ with any spinor, we derive that $D_{n}^{k}$ is modular. 


\subsection{Refinements}

The category $D_{n}^{k}$ has an invertible object $J$ of order 2 , whose twist coefficient and quantum dimension are equal to 1 . It was shown in [6] that any such modular category provides an invariant of a 3-manifold $M$ equipped with a first $\mathbb{Z}_{2}$-cohomology class. More precisely, the object $J$ defines a grading in the category, which coincides with the $\mathbb{Z}_{2}$-grading used above. For a closed 3manifold $M$, any $h \in H^{1}\left(M, \mathbb{Z}_{2}\right)$ can be represented by a sublink of a surgery link for $M$ belonging to the kernel of the linking matrix modulo 2 . The invariant of a pair $(M, h)$ is then defined by putting 1 -graded Kirby colors on this sublink and 0-graded ones on the other components. This construction can be extended to manifolds with boundary (see [3]) and leads to a cohomological TQFT (compare [11]).

Proposition 3.1 The category $D_{n}^{k}$ leads to a cohomological TQFT. The dimension of the TQFT module associated with a pair $\left(\Sigma_{g}, h\right), h \in H^{1}\left(\Sigma_{g}\right)$, is given by the following formulas. For $h \neq 0$,

$$
\operatorname{dim} V\left(\Sigma_{g}, h\right)=\frac{\langle\Omega\rangle^{g-1}}{4^{g}} \sum_{\lambda \in \Gamma \backslash \Gamma_{1}}(\operatorname{dim} \lambda)^{2-2 g}
$$

where $\Gamma_{1}=\left\{\lambda \in \Gamma: \lambda_{1}=k, \lambda_{1}^{\vee}<n\right\}$. For $h=0$,

$$
\operatorname{dim} V\left(\Sigma_{g}, h\right)=\frac{\langle\Omega\rangle^{g-1}}{4^{g}}\left(\sum_{\lambda \in \Gamma \backslash \Gamma_{1}}(\operatorname{dim} \lambda)^{2-2 g}+4^{g} \sum_{\lambda \in \Gamma_{1}}(\operatorname{dim} \lambda)^{2-2 g}\right)
$$

Proof In [6, Theorem 5.1] Blanchet give a refined Verlinde formula for cohomological TQFT's. In our case, $J$ acts on $\Gamma$ as follows:

$$
J \otimes\left(\lambda_{1}, \lambda_{2}, \ldots, \lambda_{n}\right)=\left(2 k-\lambda_{1}, \lambda_{2}, \ldots,-\lambda_{n}\right)
$$

This is because, $\lambda \otimes J$ is simple and it contains the object from the right hand side of the above formula by classical representation theory.

Therefore, we have only two cases. If $\lambda \in \Gamma \backslash \Gamma_{1}$, then $\# \operatorname{orb}(\lambda)=2,|\operatorname{Stab}(\lambda)|=$ 1. If $\lambda \in \Gamma_{1}$, then $\# \operatorname{orb}(\lambda)=1,|\operatorname{Stab}(\lambda)|=2$. The result follows by the direct application of the Blanchet formula. 


\section{Relation with quantum groups}

We show that our orthogonal modular categories are equivalent to the quantum group theoretical ones. Further, we compare results about refinements and level-rank duality.

\subsection{Equivalence}

Let us call two modular categories equivalent if there exists a bijection between their sets of simple objects providing an equality of the corresponding colored link invariants. This implies that the associated TQFT's are isomorphic (see $[13$, III, 3.3]).

Theorem 4.1 i) The category $B_{n}^{k}$ is equivalent to the modular category defined for $U_{q}\left(B_{n}\right)$ at a $(4 n+4 k)$ th root of unity $q$.

ii) The category $D_{n}^{k}$ is equivalent to the modular category defined for $U_{q}\left(D_{n}\right)$ at a $(2 n+2 k-2)$ th root of unity $q$.

Proof The construction of modular categories from quantum groups is given in [8]. Let us recall the main results.

Let $\mathfrak{g}$ be a finite-dimensional simple Lie algebra over $\mathbb{C}$. Let $d$ be the maximal absolute value of the non-diagonal entries of its Cartan matrix. Let us denote by $C$ the set of the dominant weights of $\mathfrak{g}$. We normalize the inner product ( | ) on the weight space, such that the square length of any short root is 2 . Finally, we denote by $\beta_{0}$ the long root in $C$.

The quantum group $U_{q}(\mathfrak{g})$ at a primitive root of unity $q$ of order $r$ provides a modular category if $r \geq d h^{\vee}$, where $h^{\vee}$ is the Coxeter number. This modular category has the following set of simple objects.

$$
C_{L}=\left\{x \in C:\left(x \mid \beta_{0}\right) \leq d L\right\}
$$

Here $L:=r / d-h^{\vee}$ is the level of the category.

i) Let $\mathfrak{g}=B_{n}$. Then we have $\beta_{0}=(1,1,0, \ldots, 0)$ in the basis chosen in the appendix, $d=2$ and $h^{\vee}=2 n-1$. For $r=4 n+4 k, C_{L}$ is in bijection with the set $\Gamma=\left\{\lambda: \lambda_{1}+\lambda_{2} \leq 2 k+1\right\}$ of simple objects of $B_{n}^{k}$. Moreover, this 
bijection induces an equality of the colored link invariants due to the result of Le-Murakami [9].

iI) Let $\mathfrak{g}=D_{n}$. Then $\beta_{0}=(1,1,0, \ldots, 0)$ in the basis chosen in the appendix, $d=1$ and $h^{\vee}=2 n-2$. For $r=2 n+2 k-2, C_{L}$ coincides with the set of simple objects of $D_{n}^{k}$. The claim follows then as above from [9].

\subsection{Refinements}

Cohomological refinements in categories obtained from quantum groups were studied in [11]. For type $D$, Le and Turaev consider cohomology classes with coefficients in $\mathbb{Z}_{4}$ or $\mathbb{Z}_{2} \times \mathbb{Z}_{2}$. The statements about existence of spin refinements in modular categories of type $B$ and about $\mathbb{Z}_{2}$-cohomological refinements for type $D$ seem to be new.

\subsection{Level-rank duality}

It was shown in [4] that the categories $B^{n,-k}$ and $D^{n, k}$ have their level-rank dual partners. For quantum groups this means the following. Let us denote by $\tilde{D}^{n, k}$ the modular category for $U_{q}\left(D_{n}\right)$ at a $(2 n+2 k-2)$ th root of unity quotiented by spinors and the action of the transparent object $2 k$. Then $\tilde{D}^{n, k}$ is isomorphic to $\tilde{D}^{k, n}$. The isomorphism is given by sending $v$ to $-v^{-1}$ and by 'transposing' the partitions. (It is helpful to use the geometric approach to modularization functors in order to construct the isomorphism.) In the odd orthogonal case this duality does not exist on the quantum group level, because the corresponding quotients can not be constructed. Transparent objects have twist coefficients $(-1)$.

Unfortunately, this level-rank duality between 0-graded parts of $B_{n}^{k}$ and $D_{n}^{k}$ does not extend to the full categories. Even the cardinalities of the sets of simple objects in $B_{n}^{k}$ and $B_{k}^{n}$ as well as in $D_{n}^{k}$ and $D_{k}^{n}$ are different in general. This suggests the existence of bigger categories with more symmetric sets of objects admitting level-rank duality. One possibility to construct them would be to take the 0-graded part of $B_{n}^{k}$ and to add two different spin representations using the $B_{n}$ and $B_{k}$ weight systems. This will be studied in the forthcoming paper with C. Blanchet. 


\section{Appendix}

\subsection{Odd orthogonal case}

Let $\left\{e_{i}\right\}_{i=1,2, \ldots, n}$ be the standard base of $\mathbb{R}^{n}$ with the scalar product $\left(e_{i} \mid e_{j}\right)=$ $2 \delta_{i j}$. Any weight of $B_{n}$ has all integer or all half-integer coordinates in this base. We write $\lambda=\left(\lambda_{1}, \ldots, \lambda_{n}\right)$ if $\lambda=\sum_{i=1}^{n} \lambda_{i} e_{i}$. Any weight $\lambda$ with $\lambda_{1} \geq$ $\lambda_{2} \geq \ldots \geq \lambda_{n} \geq 0$ is a highest weight of an irreducible representation of $B_{n}$ or a dominant weight. The half sum of all positive roots of $B_{n}$ we denote by $\rho=(n-1 / 2, n-3 / 2, \ldots, 1 / 2)$.

Let us consider the quantum group $U_{q}\left(B_{n}\right)$, where $q=v^{2}$ is a primitive $(4 n+$ $4 k$ ) th root of unity. The set of simple objects (or dominant weights) of the corresponding modular category is $\Gamma=\left\{\lambda: \lambda_{1}+\lambda_{2} \leq 2 k+1\right\}$, where $\lambda$ is a highest weight of $B_{n}$ [8]. The quantum dimension of a simple object $\lambda \in \Gamma$ is given by the following formula:

$$
\begin{gathered}
\operatorname{dim} \lambda=\frac{1}{\psi} \sum_{w \in W} \operatorname{sn}(w) v^{2(\lambda+\rho \mid w(\rho))} \\
\psi=\sum_{w \in W} \operatorname{sn}(w) v^{2(\rho \mid w(\rho))}=\prod_{\text {positive roots } \alpha} v^{(\rho \mid \alpha)}-v^{-(\rho \mid \alpha)}
\end{gathered}
$$

Here $W=\mathbb{Z}_{2}^{n} \propto S_{n}$ is the Weyl group of $B_{n}$ generated by reflections on the hyperplanes orthogonal to the roots $\pm e_{i} \pm e_{j}, \pm e_{i}$. Furthermore, for the invariant of the Hopf link, whose components are colored by $\mu, \nu \in \Gamma$, we have

$$
S_{\mu \nu}=\frac{1}{\psi} \sum_{w \in W} \operatorname{sn}(w) v^{2(\mu+\rho \mid w(\nu+\rho))} .
$$

Lemma 5.1 Let $b=\left(\frac{2 b_{1}+1}{2}, \frac{2 b_{2}+1}{2}, \ldots, \frac{2 b_{n}+1}{2}\right)$ be a dominant weight with halfinteger coordinates and $A=(k, k, \ldots, k)$, then

$$
\bigcup_{\mid}^{b}=\left.(-1)^{b_{1}+b_{2}+\ldots+b_{n}}\right|^{b} .
$$

Proof The coefficient to determine is equal to $S_{b A}(\operatorname{dim} b)^{-1}$. From $(7)$ and (8) we have

$$
\operatorname{dim} b=\frac{1}{\psi} \sum_{w \in W} \operatorname{sn}(w) v^{2(b+\rho \mid w(\rho))}
$$

Algebraic 83 Geometric Topology, Volume 3 (2003) 


$$
S_{b A}=\frac{1}{\psi} \sum_{w \in W} s n(w) v^{2(b+\rho \mid w(A+\rho))}
$$

Let us write $A+\rho=(k+n)(1,1, \ldots, 1)+w_{1}(\rho)$, where $w_{1} \in W$ and $s n\left(w_{1}\right)=$ $(-1)^{n(n+1) / 2}$. Then from $v^{4 n+4 k}=-1$ we have

$$
v^{2(k+n) \sum_{i}\left(b+\rho \mid \pm e_{i}\right)}=(-1)^{\left(b_{1}+b_{2}+\ldots+b_{n}+n(n+1) / 2\right)}
$$

or

$$
s n(w) v^{2(b+\rho \mid w(A+\rho))}=(-1)^{b_{1}+b_{2}+\ldots+b_{n}} s n\left(w w_{1}\right) v^{2\left(b+\rho \mid w w_{1}(\rho)\right)} .
$$

The result follows.

\subsection{Even orthogonal case}

Let $\left\{e_{i}\right\}_{i=1,2, \ldots, n}$ be the standard base of $\mathbb{R}^{n}$ with the scalar product $\left(e_{i} \mid e_{j}\right)=$ $\delta_{i j}$. Any weight of $D_{n}$ has all integer or all half-integer coordinates in this base. Any weight $\lambda=\left(\lambda_{1}, \ldots, \pm \lambda_{n}\right)$ with $\lambda_{1} \geq \lambda_{2} \ldots \geq \lambda_{n} \geq 0$ is a highest weight of an irreducible representation of $D_{n}$. The half sum of all positive roots of $D_{n}$ we denote by $\rho=(n-1, n-2, \ldots, 1,0)$.

Let us consider the quantum group $U_{q}\left(D_{n}\right)$, where $q$ is a primitive $(2 n+$ $2 k-2$ ) th root of unity and $v^{2}=q$. The set of simple objects (or dominant weights) of the corresponding modular category is $\Gamma=\left\{\lambda: \lambda_{1}+\lambda_{2} \leq 2 k\right\}$. The formulas (7) and (8) hold for the highest weights from $\Gamma$, where the Weyl group $W$ of $D_{n}$ is generated by reflections on the hyperplanes orthogonal to the roots $\pm e_{i} \pm e_{j}$. This group contains $S_{n}$. The kernel of the projection to $S_{n}$ consists of transformations acting by $(-1)$ on an even number of axes.

Lemma 5.2 Let $b=\left(\frac{2 b_{1}+1}{2}, \frac{2 b_{2}+1}{2}, \ldots, \frac{2 b_{n}+1}{2}\right)$ be a dominant weight with halfinteger coordinates, $A_{+}=(k, k, \ldots, k)$ and $i=v^{n+k-1}$, then

$$
\bigcup_{\mid}^{b} A_{+}=\left.(-1)^{b_{1}+b_{2}+\ldots+b_{n}} i^{n}\right|^{b} \text {. }
$$

Proof As before, the coefficient to determine is equal to $S_{b A_{+}}(\operatorname{dim} b)^{-1}$. We have

$$
S_{b A_{+}}=\frac{1}{\psi} \sum_{w \in W} s n(w) v^{2\left(b+\rho \mid w\left(A_{+}+\rho\right)\right)} .
$$

Algebraic 63 Geometric Topology, Volume 3 (2003) 
Let us write $A_{+}+\rho=(k+n-1)(1,1, \ldots, 1)+w_{1}(\rho)$, where $w_{1} \in W$ and $\operatorname{sn}\left(w_{1}\right)=(-1)^{n(n-1) / 2}$. Then

$$
v^{2(k+n-1)(b+\rho \mid w(1, \ldots, 1))}=(-1)^{b_{1}+b_{2}+\ldots+b_{n}+n(n-1) / 2} i^{n}
$$

for any $w \in W$. The result follows as in the odd orthogonal case.

Note that for $A_{-}=(k, k, \ldots,-k)$ an analogous statement holds:

$$
\bigcup_{\mid}^{b} A_{-}=\left.(-1)^{b_{1}+b_{2}+\ldots+b_{n}}(-i)^{n}\right|^{b}
$$

\section{References}

[1] Bar-Natan, D.: On the Vassiliev knot invariants, Topology 34 (1995) 423-472

[2] Bar-Natan, D., Le, T., Thurston, D.: Two applications of elementary knot theory to Lie algebras and Vassiliev invariants, arXiv:math.QA/0204311

[3] Beliakova, A.: Refined invariants and TQFT's from Homfly skein theory, J. Knot Theory Ramif., 8 (1999) 569-587

[4] Beliakova, A., Blanchet, C.: Modular categories of types B,C and D, Comment. Math. Helv. 76 (2001) 467-500

[5] Beliakova, A., Blanchet, C.: Skein construction of idempotents in BirmanMurakami-Wenzl algebras, Math. Annalen 321 (2001) 347-373

[6] Blanchet, C.: A spin decomposition of the Verlinde formulas for type A modular categories, arXiv:math.QA/0303240

[7] Fulton, W., Harris, J.: Representation theory, Graduate Texts in Mathematics 129, Springer-Verlag 1991

[8] Le, T.: Quantum invariants of 3-manifolds: integrality, splitting, and perturbative expansion, arXiv: math.QA/0004099

Le, T.: Integrality and symmetry of quantum link invariants, Duke Math. J. 102 (2000) 273-306

[9] Le, T., Murakami, J.: The universal Vassiliev-Kontsevich invariant for framed oriented links, Compos. Math. 102 (1996) 41-64

[10] Le, T., Murakami, J.: Kontsevich's integral for the Kauffman polynomial, Nagoya Math. J. 142 (1996) 39-65

[11] Le, T., Turaev, V.: Quantum groups and ribbon G-categories, arXiv: math.QA/0103017 
[12] Patureau, B.: Link invariant for the spinor representation of $\mathrm{SO}_{7}$, to appear in J. Knot Theory Ramif.

[13] Turaev, V.: Quantum invariants of knots and 3-manifolds, De Gruyter Studies in Math. 181994

Mathematisches Institut, Universität Basel

Rheinsprung 21, CH-4051 Basel, Switzerland

Email: Anna.Beliakova@unibas.ch

Received: 29 January $2003 \quad$ Revised: 14 August 2003

Algebraic 6 Geometric Topology, Volume 3 (2003) 